as applying to the C.I.E. system, instead of to the physics of the subject. Other points arise from Messrs. Smith, Guild and Donaldson's letter, which cannot be considered here out of considerations of space, but I hope it will be perceived that there remains material for investigation and grounds for new and broader conceptions in this subject.

Adam Hilger, Ltd., J. W. Perry.

98 St. Pancras Way,

Camden Road,

London, N.W.1.

${ }^{2}$ Smith, Guild and Donaldson, Nature, 149, 76 (1942).

2Perry, Nature, 148, 691 (1941).

${ }^{3}$ Perry, Proc. Phys. Soc., 54, 9 (1942).

- Ives, Proc. Opt. Conv., 143 (1926).

"Guild, "Discussion on Vision", 83/4 (London, 1932).

- Abney, "Colour Measurement and Mixture" (London, 1891).

'Hering, Arch. f. ges. Physiol., 60, 533 (1895).

"von Helmholtz, "Handb, d. physiol. Optik", 373 (1896).

- Guild, Proc. Opt. Conv., 94 (1926).

10 Harrison, Proc. Phys. Soc. (in the press).

\section{The Electromagnetic Mental Picture}

Recent text-books ${ }^{1}$ reflect a certain uneasiness concerning the presentation to students of 'engineer. ing' electricity. Some teachers advocate classical field theory alone, others introduce the electron theory, linking it with field theory at every stage. But the awkward unsolved problems of theory cannot then be avoided; thus, the two writers cited take quite different views concerning the induction of a current by motion of conductor and magnet respectively. These theoretical problems do not concern the designer and inventor, who needs above all a mental picture which will give a correct qualitative prediction of the functioning of any proposed apparatus. The formulæ which he will then apply for calculation may, as we know, be derived from various quite different hypotheses. Such differences as appear capable of deciding between different hypotheses are beyond the limits of error of our experimental powers ${ }^{2}$.

Useful as the field picture undoubtedly is, the literature from the time of Faraday to the present day affords numerous examples of futile experiments and discussions based upon a too literal interpretation of it. Anyone can test the fact that it may mislead the most experienced. I have asked more than a dozen highly skilled physicists and radio research men whether the inductance of a straight wire is greater or less than that of the same wire bent into a circle. Without exception they answered wrongly. The only correct answer so far came from a young chemist. In the following cases the field picture as usually stated seems to be inadequate. If a toroid solenoid is placed with its plane at right angles to that of a horseshoe magnet and with a few of its turns in the pole gap of the magnet, the field picture seiems to call for a torque on the toroid, but none on the magnet. Again, if a horseshoe magnet be rotated when placed so as to send flux through a few turns only of a short-circuited toroid, the field theory seems to call for an alternating current in the toroid, with no reactive torque on the magnet. It is interesting to consider also the effect of removing to a distance that part of the toroid which is outside the field of the magnet.

Radio is now perhaps the most important, and certainly the most progressive branch of electrical engineering, and here the particle picture is essential in vacuum tube discussion. Why not base the consideration of capacity, inductance and transmission upon it as well ? Historical piety should not lead us to burden the beginner's mind with the difficulties and artificialities of the magnetic field picture, evidenced in the treatment of open and frame aerials, or of the fields near a transmitting antenna. If radio phenomena are dealt with on the lines given in my letter of August 16, 1941, the resulting mental picture of phenomena is at east as penetrating and illuminating as that given by field theory.

Thatch End,

H. STAFFoRd HATFIELD.

Hildersham, Cambs. Jan. 24.

1 e.g., Zeleny, "Elements of Electricity and Magnetism" (New York, 1935); Cullwick, "Fundamentals of Electricity and Magnetism" (Cambridge, 1939).

"O'Rahilly, "Flectromagnetics : a Discussion of Fundamentals" (London and Cork, 1938).

\section{Occurrence of Ctenodrilus in the Pacific}

THE annelid Ctenodrilus does not seem to have been recorded outside Europe, so that its occurrence in quantity at Ladysmith Harbour, on the east coast of Vancouver Island, B.C., is perhaps worthy of note. A small artificial lagoon was constructed there last summer in connexion with work on oyster culture in which the temperature of the water rose abnormally high. Material collected with a towed plankton net in this lagoon in August contained large numbers of Ctenodrilus serratus (O. Schmidt) which has probably been swept off the eel-grass and other vegetation growing there.

Ctenodrilus is of some special interest because divergent views have been held from time to time as to its taxonomic position. It is now generally regarded as a polychæte and placed among the Cirratulidæ.
Marine Biological Station, Nanaimo, B.C. Jan. 2.

\section{Berkeley.}

\section{British Zoologist Prisoners of War in Europe}

A FELLOW of this Society resident in a country at present under German control has generously offered to help British zoologists and entomologists who are prisoners of war in Germany or the enemy-occupied countries of Europe by correspondence and the loan of literature and such other means as are within his power.

The Royal Entomological Society of London is in regular communication with this fellow and invites information regarding zoologists and entomologists known to be prisoners of war in Europe. Exact details of name and address are the only essentials required, and it is emphasized that the Society has no means of transmitting messages to prisoners except through the service organized by the British Red Cross. Information may be sent to the Registrar, Royal Entomological Society of London, 41 Queen's Gate, London, S.W.7.

Francis J. Griffin (Registrar).

Royal Entomological Society of London. 